\title{
Innovative and propagable translational research model established for cell-based therapy at Chinese PLA General Hospital
}

\author{
Weidong Han \& Xiaobing Fu* \\ Institute of Basic Medicine/Bio-therapeutic Department, Chinese PLA General Hospital, Medical College of PLA, Beijing 100853, China
}

Citation: Han, W., and Fu, X. (2016). Innovative and propagable translational research model established for cell-based therapy at Chinese PLA General Hospital. Sci China Life Sci 59, 1063-1067. doi: 10.1007/s11427-015-4869-5

The use of translational medicine in Chinese hospitals appears to have become easier over the last decade. Products developed in the laboratory can sometimes be applied in the clinic under life-saving circumstances. Arteannuin is one of the most successful examples of translation during the past decade in China, having been used to treat more than one million patients with malaria. However, several approaches developed in the laboratory, with proven benefits to patients, were subsequently aborted because of the lack of a feasible translation model. The China Food and Drug Administration (CFDA) was established in 2003 and plays an important role in ensuring the safe application of food and drugs. However, the process of applying to the CFDA is tedious and labor-intensive, resulting in the suffocation of clinical translations of scientific achievements. It is therefore essential to establish a feasible and efficacious translation routine and a clinical translational base in Chinese hospitals. The ultimate aim of translational research in Chinese hospitals is to provide treatments to fill gaps left by current proven interventions for human diseases.

Pilot clinical research in the field of cell therapy, including immune cell-based tumor therapy and stem cell-based tissue repair and regeneration, is growing rapidly worldwide. The accumulation of promising clinical data implies that cell-based disease therapy can benefit patients even after failure of already-proven approaches. Cell-based therapy has the potential to solve what have traditionally been regarded as impossible problems. However, most studies have

*Corresponding author (email: fuxiaobing@vip.sina.com) been small, single-arm trials, and no firm overall conclusions can be drawn from such studies regarding the efficacy of cell therapy relative to other drugs or whether the addition of cell therapy provides any benefit over the use of other therapeutics alone. Large-scale and large-sample clinical studies are therefore needed to verify the value of cell-based therapy and determine which subpopulations are most likely to benefit from it.

The clinical application of cell-based treatments has rapidly developed over the past five years, especially in terms of adoptive immunotherapy for tumors. In most cases, cell therapy carried out in Chinese hospitals is approved and licensed by the local management bureaus; however, the current management and application model for cell therapy in most hospitals does not favor the objective clinical verification of cell-based disease interventions. Company-initiated and commercialized models of cell therapy are not advised because of the inevitable potential for abuse. In addition, the preparation of cells by low-skilled individuals and the lack of quality control in some cases represent extremely serious problems that pose barriers to the implementation of this new and promising approach. Cell therapy, especially immune cell-based and adult stem cell-based therapies, thus remain in the research stage and are not yet routine or CFDA-proven approaches for disease treatment.

The development and establishment of an efficient translational research model and a feasible routine will promote the clinical development of cell therapy. The lack of an efficient translational model for cell therapy in most Chinese hospitals will inevitably lead to the abuse or undue clinical 
use of cell therapy, or conversely to the refusal of its use, even in patients with an urgent need for alternative treatments. The best way to develop and establish a feasible translational research model is a prominent question in Chinese hospitals, especially in research-oriented hospitals. The ideal model would benefit not only large-sample clinical studies, but also small-scale clinical trials of newly developed cellular therapeutic strategies. We therefore report on our eight-year translational research practice at Chinese PLA General Hospital, the first research-oriented hospital in China.

Prior to 2005, our team focused on laboratory research and mainly comprised scientists, researchers, and technicians. We subsequently began to develop cell-based tumor immunotherapy and stem cell-based sweat gland regeneration and diabetic treatments. We established clinical-grade immune cell culture and preparation systems, including those for patient-specific dendritic cells, cytokine-induced killer cells, natural killer cells, and autologous and umbilical mesenchymal stem cells. A large cell-processing center (CPC) was established, and a series of cellular criteria covering phenotype and magnitude, the safety detection index, and clinical application guidelines were drawn up and refined by collaboration between laboratory staff members and physicians with different clinical backgrounds. The inclusion of a cell therapy-associated ethicist and data collectors in the team allowed for the establishment of a patient follow-up system. Similar to the cell therapy model presently employed in most Chinese hospitals, we initiated a CPC-based cell therapy translational research model and collaborated widely with several traditional clinical departments, including hematology, oncology, respiratory medicine, gastroenterology, gynecology, trauma, and plastic surgery. From April 2007 to October 2011, our CPC performed almost 2,000 cytokine-induced killer cell infusions in more than 600 patients with tumors and mesenchymal stem cell-reprogrammed sweat gland transplantations in approximately 30 patients with severe skin wounds. Although our CPC maintained relatively complete cytological data, its integrity and uniformity were inferior to the clinical data collected by physicians from our collaborating departments. Furthermore, the therapeutic regimens often contradicted the basic principles of cell treatment. For example, chemotherapeutic agents or glucocorticoids were applied within one week of immune cell transfusion. Overall, fewer than 50 cases with integrated laboratory and clinical data were available for analysis (Yang et al., 2012; Lu et al., 2012; Wang et al., 2013; Sheng et al., 2009). Our five-year practice revealed that this CPC-centered and multidisciplinary collaborative translational approach provided a high-input, low-output model, at least under the current conditions of Chinese hospitals. The lack of success of this model could be attributed to several factors:

- Patients were scattered among different clinical departments, and unified management was therefore lacking.
- The understanding and objectives of physicians from different clinical departments markedly differed.

- There was a lack of knowledge of and in-depth insight into the diseases and corresponding therapeutic principles among most of the laboratory researchers, leading to an excessively idealized, impractical design for the clinical application of cell therapy.

- The requirements for cell-based therapy were established by physicians from different clinical departments, and the lack of a consistent and systematic intervention design led to problems in evaluating the efficacy of cell intervention regimens.

- Minor side effects of cell infusions were not always reported.

- Data collection and follow-up by physicians were often incomplete and/or inappropriate.

- The academic benefits were required to be balanced among physicians from different clinical departments, resulting in a lack of enthusiasm regarding coordination with laboratory researchers.

The above-mentioned CPC-based translational model was thus largely unsuitable for clinical cell therapy research in Chinese hospitals, and an improved model that bridges the gap between the laboratory and clinic is needed. The establishment of a consolidated management system that favors the exchange of knowledge between laboratory scientists and clinical physicians is a prerequisite for the progress of clinical cell therapy-based translational research.

We subsequently adjusted our CPC-centered model to a patient-centered translational (PCT) research model. This involved the creation of an integrated translational medicine research team comprising a laboratory researcher, technicians, physicians, nurses, a statistician, and an ethics and law advisor. In April 2010, a special translational medicine research ward with a one-day cell-infusion room and 35 inpatient beds was established in the internal medicine building of our hospital. The team was led by a chief scientist from the Institute of Basic Medicine of PLA General Hospital. A data collection office and an outpatient service specializing in cell therapy were also set up. A new translational medicine research management structure was developed, in which the chief scientist is in charge of the overall translational project setting, overall coordination and staff recruitment, task allocation, and administrative management. This role was designed to be fulfilled by an influential academician with an excellent clinical education background in the cell biology and cell therapy fields in China. The executive director is also the principal investigator; this individual should not only be a clinical expert, but should also have adequate long-term research experience in molecular and cell biology and cell therapy to allow him or her to be in charge of cell-based clinical translation. The appointed chief medical officer (CMO) is responsible for patient management and cell preparation, and the chief technician officer (CTO) is responsible for quality control management. The CMO is the main person responsible for the design of systematic therapeutic approaches for patients and the main 
clinical problem solver. The research and development (R\&D) group is an indispensable component of the team with respect to clinical sample treatment and special index detection. The chief R\&D officer and the CTO take joint charge of developing new strategies based on clinical requirements.

This novel organizational structure of a PCT model provides unified management possibilities that allow us to revise the content and endpoints designed for large-sample clinical evaluations of the efficacy of cell therapy. Such revisions include changes in the measurements of the response and progression-free periods, establishment of patient selection strategies, and identification of optimal combination regimens. This model also allows us to randomly group patients into cohorts to investigate the optimal opportunities, frequencies, and cycles of cell therapy interventions. Finally, the model enables us to identify the optimal interventions for adoptive immunotherapy by comparison among different tumors and examine the clinical safety and potential concerns of cell therapy through long-term observations.

This new model has currently been operational for 18 months. During this period, we have established an integrated tumor database of 1,000 patients. This database includes each patient's complete therapeutic history, status at the time of cell therapy enrollment, transfusion reactions to immune cells, other treatments, and data on regular systematic efficacy evaluations. The tumor types include advanced leukemia, lymphoma, multiple myeloma, newly diagnosed and advanced lung cancer, digestive tract tumors, liver cancer, pancreas cancer, and chemotherapy-refractory ovarian tumors. Therapeutic regimens other than adoptive immune cell therapy were comparable among patients with the same tumor type and identical disease stages, which was not the case in our previous CPC model. Analysis of our 18-month laboratory-clinic database suggested that the addition of immunotherapy (repeated infusion of autologous immune cells after ex vivo activation) could effectively inhibit the progression of various advanced tumors in $20 \%-50 \%$ of patients. Intriguingly, immune cell transfusion appeared to block lymph node pathway-mediated tumor metastasis rather than blood pathway-mediated metastasis. Immune cell infusion generally improved the debilitated status of patients with terminal cancer and effectively ameliorated the adverse reactions associated with chemotherapy. However, immunotherapy appeared to be ineffective in patients with sarcoma.

In contrast to previous reports, immune cell infusion is not without side effects. In rare cases, it may even result in life-threatening complications that require timely recognition and treatment. We summarized the rare adverse reactions among approximately 4,000 immune cell infusions in nearly 1,000 patients. Additionally, in our database analysis, we preliminarily identified the independent treatment value of adoptive immunotherapy for chemotherapy-refractory advanced B-cell lymphoma. As noted above, this newly established approach represents a highly effective model for cell-based clinical translational research. We believe that expansion of our database will allow for further significant conclusions based on immunotherapy-related clinical research.

This model may promote the rapid transfer of clinical concerns into laboratory investigations as well as the return of subsequent research results for clinical assessment. For example, with respect to the optimal frequency of adoptive immune cell infusion for tumor therapy, laboratory research determined that discontinuous intervention was preferable to continuous intervention. This was confirmed by further clinical data analysis.

This model could provide a barrier-free and rapid clinical translational platform for new cell therapy strategies through the seamless integration of $R \& D$ and clinical groups. In September 2012, our R\&D group developed a tumor antigen-specific target adoptive immunotherapy system derived from a newly generated chimeric antigen receptor (CAR) molecule, and our CTO-led group prepared clinical-grade CAR-modified $\mathrm{T}$ cells. This system was subsequently introduced to our translational ward. In less than one year, we had formally registered and initiated six CAR-modified T-cell (CART) clinical trials: CART-19, CART-20, CART-33, CART-138, CART-EGFR, and CART-HER2 (Table 1). In addition, novel therapeutic immune cells, namely natural killer $\mathrm{T}$ cells, were developed and prepared by our R\&D and CTO-led groups and have been entered into clinical assessment (Table 1).

For clinical physicians previously restricted to traditional treatment strategies, this professional translational medicine ward provides a creative environment that allows them to consider other available investigational agents based on individual patient requirements. Inspired by limited clinical data from a small-sample clinical trial and the underlying experimental research completed by a team from John Hopkins University, our CMO hypothesized that a low-dose demethylating agent may sensitize refractory tumor cells to chemotherapy and adoptive immunotherapy. The CMO subsequently designed and initiated a clinical trial evaluating the use of epigenetic therapy-primed immune cell transfusion and/or a chemotherapeutic agent in patients refractory to chemotherapy and adoptive immunotherapy (Table 1). More than 100 patients have been enrolled in this trial to date. The initial clinical results indicate that patients with advanced primary hepatic cancer may benefit from this regimen.

In addition to the beneficial clinical effects of this new translational research model, this laboratory-clinical framework also provides an active environment for research. In many cases, rare laboratory characteristics of individual patients can be observed by researchers, and direct researcher-patient contact can trigger thinking patterns and approaches outside of the traditional clinical concepts 
Table 1 Clinical cell-therapy trials initiated by our translational team from February 2013

\begin{tabular}{|c|c|c|c|}
\hline Gov ID of clinical trials & Brief title & $\begin{array}{l}\text { Overall } \\
\text { status }\end{array}$ & Completed cases \\
\hline NCT01864889 & $\begin{array}{l}\text { Treatment of relapsed and/or chemotherapy- } \\
\text { refractory B-cell malignancy by CART19 }\end{array}$ & Recruiting & 9 (B cell acute lymphocytic leukemia) \\
\hline NCT01735604 & $\begin{array}{l}\text { Genetically-engineered lymphocyte therapy to treat } \\
\text { patients with lymphoma that is resistant or refracto- } \\
\text { ry to chemotherapy }\end{array}$ & Recruiting & $\begin{array}{l}11 \text { ( } 10 \text { patients with diffuse large B-cell lymphoma } \\
\text { and } 1 \text { patient with CD20-positive multiple myeloma) }\end{array}$ \\
\hline NCT01864902 & $\begin{array}{l}\text { Treatment of relapsed and/or chemotherapy- } \\
\text { refractory CD33-positive acute myeloid leukemia } \\
\text { by CART-33 (CART33) }\end{array}$ & Recruiting & 1 (acute myeloid leukemia) \\
\hline NCT01886976 & $\begin{array}{l}\text { Treatment of chemotherapy-refractory multiple } \\
\text { myeloma by CART-138 }\end{array}$ & Recruiting & 5 (multiple myeloma) \\
\hline NCT01869166 & $\begin{array}{l}\text { Treatment of chemotherapy-refractory epidermal } \\
\text { growth factor receptor (EGFR)-positive advanced } \\
\text { solid tumors (CART-EGFR) }\end{array}$ & Recruiting & 14 (lung cancer) \\
\hline NCT02259556 & $\begin{array}{l}\text { CD3-directed chimeric antigen receptor } \mathrm{T} \\
\text { (CART30) in relapsed and refractory CD30 positive } \\
\text { lymphomas }\end{array}$ & Recruiting & 13 (Hodgkin’s Lymphoma) \\
\hline NCT01935843 & $\begin{array}{l}\text { Treatment of chemotherapy-refractory human epi- } \\
\text { dermal growth factor receptor-2 (HER-2)-positive } \\
\text { advanced solid tumors (CART-HER-2) }\end{array}$ & Recruiting & 0 \\
\hline NCT01801852 & $\begin{array}{l}\text { Autologous natural killer } \mathrm{T} \text { cell infusion for the } \\
\text { treatment of cancer }\end{array}$ & Recruiting & 6 (sarcoma or sarcoma-like cancer) \\
\hline NCT01799083 & $\begin{array}{l}\text { Lower dose decitabine-based therapy in patients } \\
\text { with refractory and/or chemotherapy-resistant solid } \\
\text { tumors or B cell lymphomas (CIK) }\end{array}$ & Recruiting & $\begin{array}{l}178 \text { (various solid tumors including } 26 \text { primary } \\
\text { hepatocyte cancers) }\end{array}$ \\
\hline
\end{tabular}

adopted by most physicians. For example, although physicians were only aware of biclonal or biphenotypic multiple myeloma, a novel triphenotypic type of multiple myeloma was identified in our translational medicine ward as a result of queries initiated by laboratory staff members based on clinicians' descriptions and discussions (Liu et al., 2013).

Our eight-year experience in translational research suggests that this ward-patient-centered and joint scientist-physician model (also referred to as a "working hand-inhand model") can help to bridge the therapeutic gaps left by currently proven interventions for human diseases. The significance of translational research in hospitals lies in its ability to make the seemingly impossible possible. This represents a highly efficient model for translational medicine that is especially suitable for Chinese research-oriented hospitals.

Based on our limited experience, we suggest that daily researcher-clinician meetings form a core aspect of this novel translational research model, together with periodic reciprocal education programs. The participating members include scientists, physicians, technicians, and nurses. Discussions encompass medical safety, clinical research, and patient responses, among other topics. These meetings suggest to the researchers which clinical questions should be investigated in the laboratory, and in turn alert physicians to the parameters worthy of more attention and careful recording. Although our current team includes few physician scientists, periodic reciprocal education and information exchange between scientists and physicians with different clinical backgrounds can compensate for this lack. Scientists encountering clinical situations or individual patients are able to approach problems from new angles.
Translational medicine should not be considered to be a special discipline, but rather a model that can organically link multiple disciplines and coordinate talents from different backgrounds to achieve a single goal. The coordination of these basic and clinical research backgrounds is a priority for the implementation of translational medicine. Established translational models in Western counties may be not suitable for Chinese hospitals because an efficient model must take several factors into account, including cultural and social differences. This ward-patient-centered and laboratory-clinic integrative translation model established at Chinese PLA General Hospital may be suitable for extension to other Chinese research-orientated hospitals. However, duplication of this model in most Chinese hospitals still has a long way to go; notably, biotherapeutic translation is only the first step. Nevertheless, to realize this goal, we should take note of the Chinese proverb alleging that "constant dropping wears the stone".

Compliance and ethics The author(s) declare that they have no conflict of interest.

Acknowledgements This work was supported by the National Natural Science Foundation of China (81230061, 81121004, and 81230041), the Military Medical Foundation (AWS11J008), and the National Basic Research Program of China (2012CB518105).

Liu, Y., Zhang, Y., and Han, W. (2013). Triphenotypic multiple myeloma expressing kappa or lambda light chain or both. Br J Haematol 160, 4.

Lu, X.C., Yang, B., Yu, R.L., Chi, X.H., Tuo, S., Tuo, C.W., Zhu, H.L., Wang, Y., Jiang, C.G., Fu, X.B., Yang, Y., Liu, Y., Yao, S.Q., Dai, H.R., Cai, L.L., Li, B.J., and Han, W.D. (2012). Clinical study of au- 
tologous cytokine-induced killer cells for the treatment of elderly patients with diffuse large B-cell lymphoma. Cell Biochem Biophys 62, 257-265.

Sheng, Z.Y., Fu, X.B., Cai, S., Lei, Y.H., Sun, T.Z., Bai, X.D., and Chen, M.L. (2009). Regeneration of functional sweat gland-like structures by transplanted differentiated bone marrow mesenchymal stem cells. Wound Repair Regen 17, 427-435.

Wang, Y., Bo, J., Dai, H.R., Lu, X.C., Lv, H.Y., Yang, B., Wang, T., and
Han, W.D. (2013). CIK cells from recurrent or refractory AML patients can be efficiently expanded in vitro and used for reduction of leukemic blasts in vivo. Exp Hematol 41, 241-252.

Yang, B., Lu, X.C., Yu, R.L., Chi, X.H., Liu, Y., Wang, Y., Dai, H.R., Zhu, H.L., Cai, L.L., and Han, W.D. (2012). Repeated transfusions of autologous cytokine-induced killer cells for treatment of haematological malignancies in elderly patients: a pilot clinical trial. Hematol Oncol 30, 115-122.

Open Access This article is distributed under the terms of the Creative Commons Attribution License which permits any use, distribution, and reproduction in any medium, provided the original author(s) and source are credited. 